\title{
Búsqueda y recuperación de la literatura científica
}

\author{
José Darío Martínez-Ezquerro ${ }^{1, \S}$, Mario Enrique Rendón-Macías²
}

${ }^{1}$ Unidad de Investigación Médica en Genética Humana, Centro Médico Nacional Siglo XXI, Instituto Mexicano del Seguro Social (IMSS)

${ }^{2}$ Unidad de Investigación en Análisis y Síntesis de la Evidencia, Centro Médico Nacional Siglo XXI, Instituto Mexicano del Seguro Social (IMSS)

$\S$ Correspondencia:

jdme@ciencias.unam.mx (J.D. Martínez-Ezquerro)

\section{Introducción}

En sentido amplio, investigar involucra cualquier búsqueda y recopilación sistemática y pertinente de datos, información y hechos, así como su análisis, para aumentar el conocimiento sobre un tema determinado $(1,2)$.

La búsqueda de la literatura y la recopilación de referencias forman parte de una etapa precisa del modelo secuencial de los componentes de una investigación (2). Cabe resaltar que un aspecto fundamental durante todo el proceso de investigación involucra el conocimiento y dominio práctico de la literatura científica también llamado alfabetización informacional, que consiste en comprender la información y cómo funciona. El desarrollo de esta habilidad de investigación implica realizar y ser capaz de responder preguntas tales como ¿cuáles son las fuentes de información que tengo disponibles?, ¿cuál es su naturaleza para el contexto específico de mi interés?, ¿cómo puedo encontrar, evaluar y usar de manera efectiva y éticamente dicha información?

Aunque la búsqueda de la literatura científica y la recopilación de referencias deben estipularse claramente y ejecutarse como una etapa del proceso de investigación en este modelo secuencial, en la práctica no se lleva a cabo de forma unívoca, singular, sino en sentido plural: un conjunto de búsquedas y recopilaciones de información académica que, además de realizarse en una etapa precisa, se llevan a cabo durante todo el proceso de investigación -antes, durante y después.

Así, la alfabetización informacional nos permite explorar los temas de nuestro interés, generar ideas, seleccionar un tema que nos apasione profundizar mediante la definición de un problema o pregunta de investigación, sobre la cual buscaremos, recopilaremos y actualizaremos información académica relevante (Figura 1). 


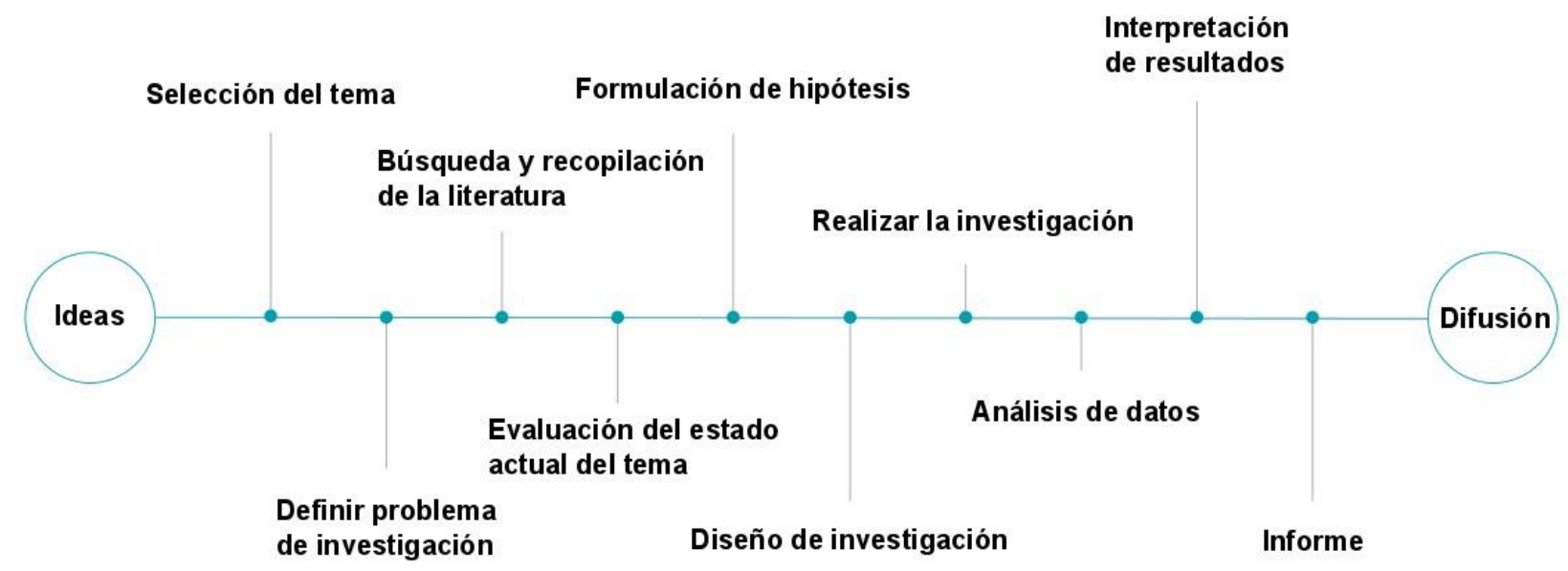

Figura 1. Ciclo dinámico de la investigación. En este modelo secuencial de la investigación se observan 10 etapas generales de una investigación (puntos azules), considerando además dos etapas pre- y post-investigación fundamentales (nodos azules) que reconocen que el surgimiento de este proceso deriva del conocimiento previo (“...on the shoulders of giants.", John of Salisbury; Isaac Newton), el proceso se completa con la difusión de la investigación y, finalmente, el ciclo se completa al unir los nodos ideas-difusión-ideas, haciendo de la investigación, un proceso dinámico.

En este capítulo se presentan las principales bases de datos de la literatura académica que contienen datos relevantes a las áreas de la salud y biomédicas. Además, presentamos estrategias de búsqueda bibliográfica para facilitar su adquisición cotidiana, sistemática, reproducible y precisa, que nos permita realizar investigaciones profundas y generar nuevo conocimiento.

\section{La búsqueda de la literatura científica}

Las búsquedas bibliográficas hacen referencia a las actividades necesarias para recopilar documentos relacionados a un tema específico y poder determinar el estado actual del conocimiento relacionado (3).

Uno de los principales lugares de consulta de la literatura publicada son las bases de datos bibliográficos en línea; éstas pueden contener artículos, resúmenes, libros y capítulos de libros, entre otros documentos de investigación. Algunas de las principales características de este tipo de bases de datos incluyen la información estructurada y ordenada en registros y campos, su indización por descriptores, enlaces al texto completo, así como una interfaz de búsqueda (3). 
Esta información estructurada -que puede variar entre las bases de datos-facilita tanto la búsqueda de información como su recuperación, a través de diversos campos: nombre de los autores, título del artículo, año de publicación, nombre de la revista en la que se publicó, volumen, número y páginas en las que se encuentra el artículo, identificador de objeto digital (digital object identifier, DOI), entre otras.

Con la asignación del código alfanumérico DOI (4) se pueden identificar, buscar y localizar diversos recursos científico-académicos: libros y revistas completos, así como edición o número individual, capítulos o artículos científicos electrónicos, así como resúmenes individuales, figuras, tablas, estructuras químicas, audios, videos, imágenes, pósters, presentaciones y software, incluso aunque el elemento digital cambie de propietario o de alojamiento en la web a un servidor distinto del alojamiento inicial. Se compone por la identificación del editor (prefijo) y un identificador del objeto (sufijo) separados por una barra “/” $(5,6)$. Por ejemplo, el DOI para un artículo de F1000Research (7) y de PeerJ (8) serían: 10.12688/f1000research.9756.1 y 10.7717/peerj.1701, respectivamente. Debido a la posible volatilidad de la información contenida en internet, la asignación de identificadores digitales como el DOI resulta útil ya que permite identificar y recuperar rápidamente el recurso electrónico que identifica, además de garantizar de manera única el acceso directo y permanente el elemento digital asignado aunque cambie su ubicación en la red. Además, garantiza la propiedad intelectual de un recurso electrónico mediante la información asociada en sus metadatos y permite la interoperabilidad entre plataformas, repositorios y motores de búsqueda.

A continuación veremos características generales de algunas de ellas. Además, mencionaremos brevemente otras posibles fuentes de búsqueda de literatura científica como preprints, redes sociales convencionales y académicas, y blogs. Posteriormente, revisaremos la estrategia de búsqueda de la literatura científica y cómo se puede estructurar en algunas de las bases de datos mencionadas previamente.

\section{Bases de datos, repositorios y motores de búsqueda bibliográficos}

A nivel mundial, diversas instituciones tienen entre sus objetivos proporcionar acceso libre y gratuito a bases de datos de la literatura biomédica. Por ejemplo, la National Library of Medicine (NLM) (9) de Estados Unidos a través de MEDLINE (10), proporciona registros procedentes de todo el mundo. A nivel regional, el Centro Latinoamericano y del Caribe de Información en 
Ciencias de la Salud, también conocido como Biblioteca Regional de Medicina (BIREME) (11), es es un centro especializado de la Organización Panamericana para la Salud (OPS)/Organización Mundial de la Salud (OMS) que coordina la Biblioteca Virtual de Salud (BVS) (12), la cual proporciona acceso - además de MEDLINE- a la evidencia científica y técnica de ciencias de la salud de los países de América Latina y el Caribe, indizada en la base de datos LILACS (Literatura Latinoamericana y del Caribe en Ciencias de la Salud) (13).

Tanto MEDLINE como LILACS, cuentan con un tesauro de vocabulario controlado de términos médicos para la indización y clasificación temática de su contenido, así como la búsqueda y recuperación de información: MeSH (Medical Subject Headings) (14) y DeCS (Descriptores en Ciencias de la Salud) (15), respectivamente. El tesauro permite el uso de una terminología común para la búsqueda y proporcionando un medio consistente y único para la recuperación de la información. En el caso de los DeCS, el vocabulario controlado es trilingüe: español, inglés y portugués, desarrollado a partir de los $\mathrm{MeSH}$, para buscar en tres idiomas y recuperar la información de manera consistente, independientemente del idioma.

Existen bases de datos bibliográficos y motores de búsqueda web de la literatura científica tanto de libre acceso -PubMed, Europe PMC y BVS-, como comerciales -Scopus, Web of Science, OVID y ProQuest-, con las que podemos buscar en bases de datos como MEDLINE y LILACS o en sus propias bases de literatura científica. Todos estos recursos de búsqueda de la literatura científica permiten filtrar la información de búsqueda -ya sea de manera directa o con la opción de búsqueda avanzada-mediante diversos criterios de selección: título, resumen, palabras clave o las tres combinadas, tema, año o intervalo de tiempo, autor, nombre de la publicación, tipo de publicación, entre otras.

Algunas de las principales bases de datos, motores de búsqueda y tesauros se presentan sintetizados en las tablas I y II.

Tabla 1. Bases de datos, repositorios y tesauros de la literatura científica.

\begin{tabular}{llc}
\hline Nombre & Sitio web & Registros \\
\hline MEDLINE & https://www.nlm.nih.gov/bsd/medline.html & 25 millones \\
Europe PMC & https://europepmc.org/ & 34.9 millones \\
LILACS & $\underline{\text { http://lilacs.bvsalud.org/es/ }}$ & 849,813 \\
SciELO & $\underline{\text { http://www.scielo.org/ }}$ & 745,182 \\
Redalyc & $\underline{\text { http://www.redalyc.org/home.oa }}$ & 613,097 \\
\hline \hline
\end{tabular}




\begin{tabular}{llc} 
PubMed & $\underline{\text { https://www.ncbi.nlm.nih.gov/pubmed/ }}$ & 29.1 millones \\
PMC & https://www.ncbi.nlm.nih.gov/pmc/ & 5.2 millones \\
Scopus & $\underline{\text { htps://www.scopus.com/ }}$ & 71 millones \\
Web of Science & https://clarivate.com/products/web-of-science/ & 151 millones \\
BVS & $\underline{\underline{\text { htp://pesquisa.bvsalud.org/portal/ }}}$ & 28.2 millones \\
\hline \hline PubMed clinical queries & $\underline{\text { https://www.ncbi.nlm.nih.gov/pubmed/clinical }}$ & $>6$ millones \\
MeSH database & $\underline{\text { https://www.ncbi.nlm.nih.gov/mesh/ }}$ & 277,539 \\
DeCS & $\underline{\text { htp://decs.bvs.br/E/homepagee.htm }}$ & 33,558 \\
\hline
\end{tabular}

Además de Google Scholar (Google académico), existen otros motores de búsqueda de la literatura científica con características distintivas que pueden consultarse para obtener panoramas generales (Tabla II).

Tabla II. Motores de búsqueda de la literatura científica

\begin{tabular}{ll}
\hline Nombre & Sitio web \\
\hline $\begin{array}{l}\text { Google Scholar } \\
\text { PubMed PubReMiner }\end{array}$ & $\underline{\text { https://scholar.google.com/ }}$ \\
GoPubMed & $\underline{\text { htps://hgserver2.amc.nl/cgi-bin/miner/miner2.cgi }}$ \\
BASE & $\underline{\text { https://www.gopubmed.org//web/gopubmed// }}$ \\
Dimensions & $\underline{\text { https://app.dimense-search.net/ }}$ \\
WorldWideScience & $\underline{\text { https://worldwidescience.org/ }}$ \\
Semantic Scholar & $\underline{\text { https://www.semanticscholar.org/ }}$ \\
CiteSeerX & $\underline{\text { http://citeseerx.ist.psu.edu/index }}$ \\
Open Knowledge Maps & $\underline{\text { https://openknowledgemaps.org/index }}$ \\
\hline
\end{tabular}

\section{Plataformas alternativas de publicación de la literatura científica}

Además de las plataformas en las que las instituciones archivan las tesis de los sustentantes que obtuvieron un grado académico de licenciatura, maestría y doctorado -por ejemplo de la UNAM http://dgb.unam.mx/index.php/catalogos/tesiunam- $\mathrm{o}$ archivos globales de tesis de acceso abierto y asignación de DOI (https://thesiscommons.org), existen otras fuentes de literatura científica distintas al modelo convencional de publicaciones post-revisión entre pares en revistas científicas. Estos modelos innovadores han surgido en respuesta a las diversas deficiencias que presenta dicho 
modelo convencional: plataformas de publicación de preprints, de artículos finales para su revisión de pares post-publicación, de redes sociales académicas, entre otras.

\section{Preprints y plataformas de revisión por pares post-publicación}

Los servidores de preprints son plataformas gratuitas y de acceso abierto que archivan e indizan manuscritos académicos -incompletos o versiones finales- antes de que reciban revisión por pares para su publicación convencional en revistas científicas. Algunos ejemplos de servidores de preprints pueden encontrarse en línea (16) incluyendo a Authorea (17), arXiv (18), bioRxiv (19), MedRxiv (20), OSF Preprints (21), Preprints (22), socArXiv (23), Zenodo (24), PeerJ Preprints (25), aunque dicho listado no incluye aquellos generados por editoriales comerciales como Nature precedings (26) y JMIR preprints (27). La publicación de preprints es muy rápida - de 24 a 48 horas-, una vez moderado que su contenido sea científico y cumpla con el ámbito de interés del servidor de preprints elegido. Además, los preprints reciben DOI, estableciendo un precedente, que a su vez permite tanto su recuperación en plataformas de búsqueda como su citabilidad, y formando parte permanente del registro científico mundial. Los lectores pueden dejar comentarios y hacer preguntas, mientras que los autores pueden actualizar su artículo con revisiones ilimitadas. Los autores conservan los derechos de autor y eligen entre varias opciones de distribuciónreutilización para que el artículo esté disponible (CC-BY, CC-BY-NC, CC-BY-ND, CC-BY-NC$\mathrm{ND}, \mathrm{CC} 0$ o no reutilización). Algunos de estos servidores facilitan el envío del preprint para su revisión por pares a las revistas científicas asociadas. Los preprints de bioRxiv, por ejemplo, son indizados por Google, Google Scholar, CrossRef, Meta, Microsoft Academic Search, y otras plataformas de búsqueda, y no están indizados en Web of Science. Una herramienta para facilitar la búsqueda de preprints es PrePubMed (28). En ella se indizan preprints de diversas plataformas alternativas: arXiv q-bio, PeerJ Preprints, bioRxiv, F1000Research, preprints.org, The Winnower, Nature Precedings y Wellcome Open Research.

A la par de estas plataformas innovadoras de publicación científica, han surgido otras dedicadas a la revisión por pares post-preprint como Prereview https://www.prereview.org y Academic Karma http://academickarma.org, y post-publicación como F1000 Research https://f1000research.com/ y The Winnower https://www.thewinnower.com, aportando al desarrollo de nuevos modelos de publicación científica gratuitos, rápidos y de acceso abierto. 


\section{Estrategias de búsqueda bibliográfica}

Con la enorme cantidad de artículos científicos publicados desde hace aproximadamente 350 años desde la aparición en 1665 de la revista científica más antigua Philosophical Transactions (29,30) junto con las otras $\sim 30,000$ revistas científicas actuales (30) y considerando que de 2015 a 2017 sólo en MEDLINE se agregaron más de 800,000 registros anualmente (31), recuperar la información científica relevante para nuestra pregunta de investigación requiere del desarrollo de una estrategia de búsqueda eficaz.

Existen distintas etapas para la búsqueda eficiente de información científica (Figura 2):

1. Planificación. La finalidad de esta etapa es clarificar los parámetros que contendrá la búsqueda de información: qué necesitamos buscar, tipo de documento que queremos recuperar, objetivo de la búsqueda, tiempo y recursos disponibles, uso de palabras combinadas mediante operadores booleanos, de proximidad, de truncamiento y los paréntesis cuando es necesario.

2. Objetivo de la búsqueda. Es vital asentar un objetivo claro al iniciar el proceso de búsqueda ya que éste servirá para dos aspectos fundamentales: a) dirigir la localización de la información mediante criterios específicos, y b) evitar que la recopilación de datos se desvíe a aspectos tangenciales que pueden estar relacionados con nuestro tema de investigación pero indirectamente asociados a la pregunta que queremos resolver, aspecto que puede impedir el mejor aprovechamiento de los recursos.

3. Pregunta de búsqueda o investigación y criterios de búsqueda. En esta etapa debemos identificar los conceptos, así como su representación mediante términos precisos y sus relaciones, generalmente en inglés y utilizando tesauros y diccionarios terminológicos. En esta etapa es importante tener un registro de las abreviaturas de las palabras clave de interés, sus sinónimos, variantes ortográficas y gramaticales, así como términos relacionados.

4. Selección de fuentes de información. Para ello, debemos conocer la cobertura documental, temporal y temática, determinar el idioma de la búsqueda así como el tipo de estudio de interés y las fuentes de información a consultar. 
Identificar conceptos

y términos clave

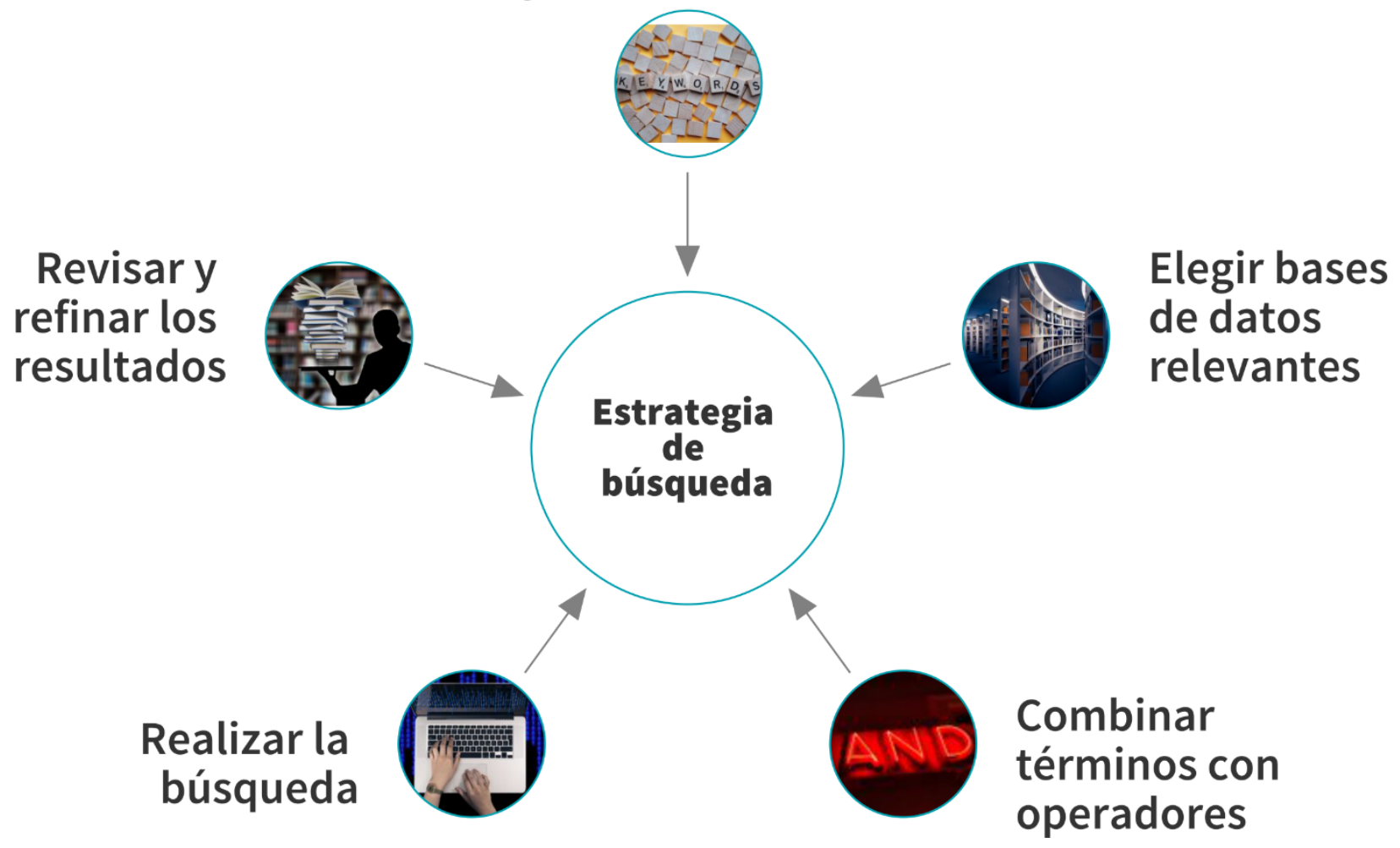

Figura 2. Estrategia de búsqueda de la literatura científica.

5. Plantear la estrategia de búsqueda (32). Los términos de búsqueda utilizan operadores booleanos -que definen la interacción entre las palabras y son $A N D, O R, N O T$ - (Figura 3), truncamientos - para cortar la palabra al principio, en medio o al final con símbolos como * o ? para reemplazar letras o caracteres, por ejemplo, medic* para medic, medicine, medical, medication-, operadores de proximidad -para indicar cercanía, adyacencia y el orden entre los términos de búsqueda near (n) o with (w) - y, de ser necesario, los paréntesis -agrupan sinónimos o términos relacionados unidos por el operador $O R$, limita y ordena la relación entre las palabras, indica el orden del procesamiento de las palabras empezando por las que se encuentran entre paréntesis-.

a. Texto libre: El uso de palabras, frases y términos de texto libre es la forma más intuitiva de búsqueda y es fácil encontrar estos componentes en el registro de cada referencia: título, autores, resumen, palabras clave; sin embargo, requiere del uso de todas las posibles formas en las que el concepto podría aparecer en la bibliografía 
b. Lenguaje controlado: Cuando la base de datos cuenta con vocabulario controlado, ésta puede incluir tesauros, encabezamientos, listas de materias o descriptores, los cuales facilitan la localización de la información independientemente de las variantes o sinónimos que puedan existir en la bibliografía. Por ejemplo, MEDLINE utiliza el tesauro de encabezados de temas médicos (Medical Subject Headings, MeSH) para indizar los artículos biomédicos incluidos en su base de datos.

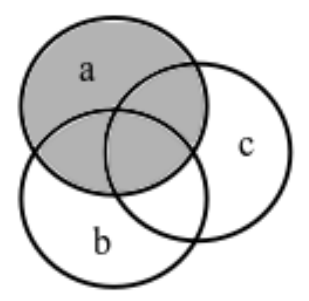

a

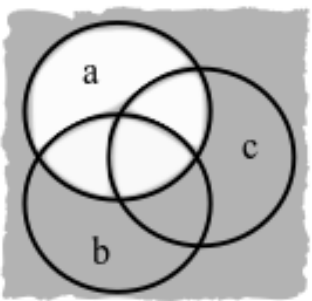

NOT a

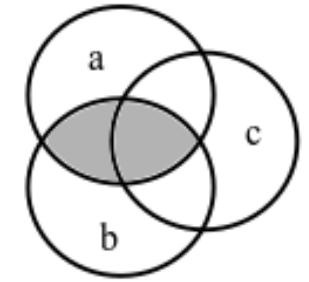

a AND b

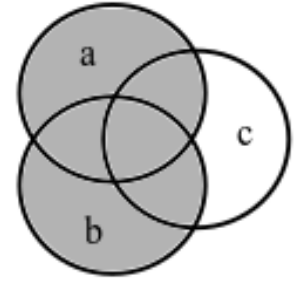

a OR b

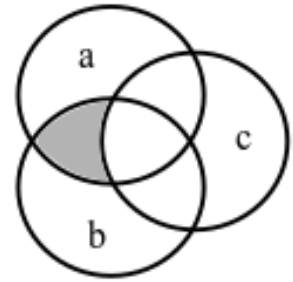

a AND b NOT c

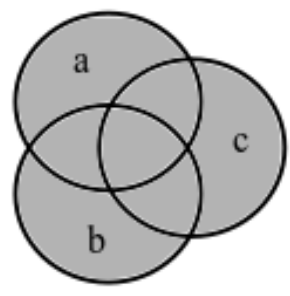

a OR b OR c

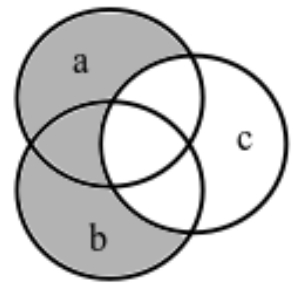

(a OR b) NOT c

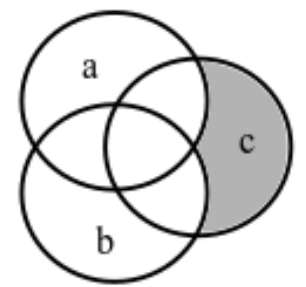

c NOT a NOT b

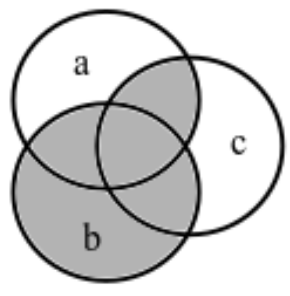

b OR (c AND a)

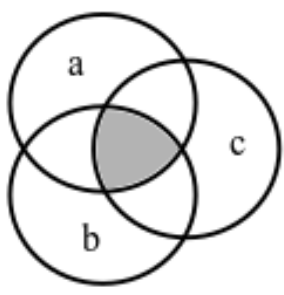

a AND b AND c

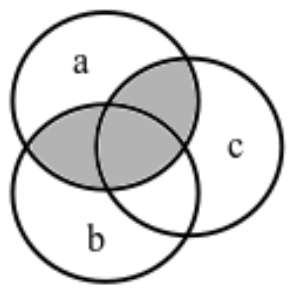

(b OR c) AND a

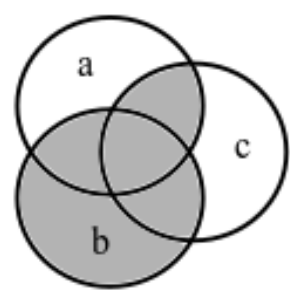

b OR c AND a

Figura 3. Representación de búsquedas de la literatura con operadores booleanos mediante diagramas de Venn. La imagen representa las formas en que se relaciona o filtra la información al buscar con operadores booleanos que condicionan su inclusión o exclusión según criterios específicos. 
Ante la propuesta de este trabajo como una guía para la actividad científica y la búsqueda de la literatura científica, es importante resaltar que diversos elementos que pueden presentarse en el desarrollo de una investigación pueden alterar los descubrimientos o resultados. Lo anterior nos demanda que revisemos y afinemos los parámetros de inclusión y exclusión de la información así como la forma de integrar los datos recopilados. Este proceso cíclico de revisión y afinación está permeado tanto por la maduración de nuestras ideas como por la necesidad de actualizar la información y aprovechar los recursos, genera una retroalimentación y reconsideración de los términos y estructuras de búsqueda avanzada, así como la posible reestructuración de la información clave (Figura 2). Por lo tanto, debemos mantener vigilancia y revisión constante en la congruencia de la información recopilada con nuestros objetivos y explorar, si es necesario, formas diversas de indagar en ella. Es decir, considerar que "ante una pregunta de investigación no hay una única estrategia ni un sólo recurso" (3).

\section{Búsqueda convencional vs. búsqueda avanzada en distintas plataformas}

Existen diferencias claras respecto a los registros que podemos recuperar al realizar las búsquedas de manera intuitiva o convencional en comparación con búsquedas estructuradas o avanzadas cuando utilizamos cualquier base de datos o motor de búsqueda.

De manera intuitiva-convencional, es común buscar títulos completos o términos de interés. En contraste, la búsqueda estructurada-avanzada nos permite limitar los resultados de la búsqueda, por ejemplo, a aquellos registros en los que aparecen tres términos en el título, en título y resumen, o en título, resumen y palabras clave, en todo el texto, así como por temporalidad.

Si además utilizan los diagramas de Venn mostrados anteriormente como guía para realizar búsquedas de literatura científica con operadores booleanos, así como comillas, paréntesis y truncamientos, poco a poco irán desarrollando herramientas de alfabetización informacional que les permitirán realizar investigaciones profundas, exhaustivas, y generar nuevo conocimiento.

En este proceso, los operadores booleanos proporcionan una forma de generar consultas precisas que producen conjuntos de resultados bien definidos y reproducibles (Figura 3). En general deben utilizarse en mayúsculas y los utilizados comúnmente son: 
AND: Encuentra documentos que contienen los términos localizados en ambos lados del operador, es decir, la intersección de ambas búsquedas. Se utiliza para reducir una búsqueda.

OR: Busca documentos que contengan cualquiera de los dos términos, es decir, la unión de ambas búsquedas. Se utiliza para ampliar una búsqueda al enlazar términos relacionados o similares.

NOT: Localiza documentos que contienen el término a la izquierda pero no el término a la derecha del operador, es decir, la resta de la búsqueda de la derecha de la búsqueda de la izquierda. Se utiliza para reducir una búsqueda.

Usualmente se procesan todos los operadores booleanos en una secuencia de izquierda a derecha. La inclusión de conceptos individuales entre paréntesis cambia esta prioridad. Los términos dentro de los paréntesis se procesan primero como una unidad y luego se incorporan a la estrategia general. Convencionalmente, los términos de búsqueda individuales separados por un espacio son combinados como si se utilizara el operador AND entre ellos. El uso de frases o términos entre comillas, aunque puede resultar útil para restringir los documentos recuperados sólo a aquellos documentos con coincidencias exactas a la cadena de texto dentro de las comillas, también puede impedir la asignación automática de términos de los términos individuales a vocabularios controlados, como los MeSH y DeCS o el organismo (taxonomía), por lo que deben utilizarse con precaución.

A continuación se presenta un esquema que visibiliza posibles estrategias de búsqueda avanzada combinando tres áreas de interés clínico: población, enfermedad e intervención [esquema PICO; población- intervención-comparador-resultado u outcome] (Figura 4). Es de esperar que con el apoyo de los diagramas de Venn presentados anteriormente (Figura 3), anticipen la información científica que será recuperada dependiendo del operador booleano utilizado para enlazar los términos elegidos. 


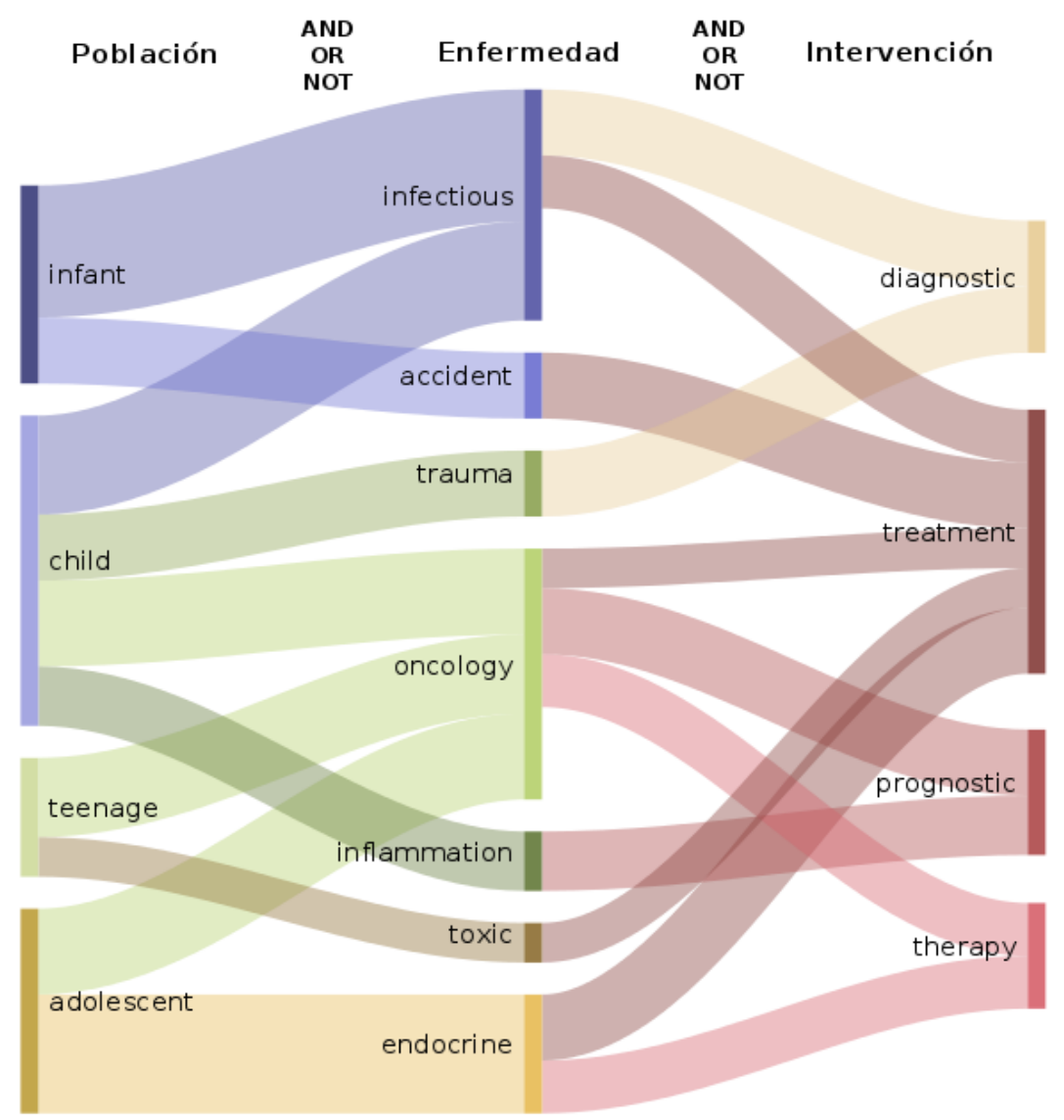

Figura 4. Estrategias de búsqueda estructurada. El diagrama de Sankey ejemplifica los flujos entre posibles estrategias de búsqueda utilizando la combinación de un término en inglés relacionado a la población de estudio, a una condición clínica y una intervención, asociados por operadores booleanos: AND, OR, NOT.

En términos prácticos, podemos anticipar que la búsqueda de nuestro tema de interés en las distintas bases de datos, utilizando la misma estrategia de búsqueda acoplada a los requerimientos de cada una de éstas, resultará no sólo en diferencias cuantitativas de los artículos registrados, sino también en los autores, instituciones, países, revistas científicas, años de publicación representados y diversas tendencias. Este nivel de análisis de la literatura científica se conoce como análisis bibliométrico y su aplicación rutinaria en toda investigación académica resulta fundamental para tratar de recuperar toda la información relevante a nuestro tema de investigación. Además, los análisis bibliométricos facilitan la toma de decisiones sobre posibles grupos académicos con los cuales establecer colaboraciones, revistas académicas en las cuales publicar o recuperar información de interés, así como definir o mantener líneas de investigación. 


\section{Recuperación de la literatura científica en texto completo}

Una vez que hemos realizado y refinado nuestras búsquedas de artículos científicos y, a pesar de que bases de datos como PubMed, Europe PMC y BVS cuentan en conjunto con millones de artículos en texto completo, de acceso libre y gratuito, por lo general nos encontraremos con una gran cantidad de artículos a cuyos textos completos no podremos tener acceso dado que se encuentran detrás de un muro de pago o paywall. En algunas ocasiones, podremos acceder a ellos a través de universidades u otras instituciones que pagan suscripciones a revistas -por lo general son paquetes de revistas de una editorial particular-o a bases de datos comerciales como Scopus y Web of Science.

$\mathrm{Al}$ respecto del esquema actual de bloqueo del conocimiento científico por fines de lucro, la Unión Europea ha iniciado la iniciativa Plan $S$ (https://www.coalition-s.org/) en apoyo a la ciencia abierta (open science) sobre la publicación instantánea de artículos en revistas o plataformas de acceso abierto y gratuito de toda la investigación financiada por fondos públicos o público-privados, de manera obligatoria a partir de 2020. Mientras ésta y otras iniciativas se aplican en Europa y eventualmente en los demás países, incluyendo México -si la cordura lo permite- ¿qué podemos hacer para recuperar el texto completo de los artículos de nuestro interés?

Aprovechando la conectividad que nos proporciona internet, existen diversos recursos en línea que podemos aprovechar para recuperar los artículos en texto completo como Google Scholar que proporciona enlaces a la versión de acceso abierto del recurso bibliográfico. Además, Unpaywall (33) es una extensión para el navegador de código abierto que permite encontrar 22,929,126 artículos de acceso abierto de más de 50,000 editoriales y repositorios mediante el uso de oaDOI -servicio abierto en línea que determina el estado de acceso abierto para 67 millones de artículos. Por otro lado, podemos buscar de manera gratuita directamente en las revistas científicas de acceso abierto en el directorio DOAJ (Directory of Open Access Journals, https://doaj.org/) (34) y en los repositorios académicos de acceso abierto en el directorio OpenDOAR (Directory of Open Access Repositories, http://v2.sherpa.ac.uk/opendoar/) (35). Otra opción es la red social para científicos e investigadores, Research gate https://www.researchgate.net/, cuyo principal objetivo es conectar al mundo de la ciencia y hacer que la investigación sea abierta para todos. Actualmente cuenta con 15 millones de miembros alrededor del mundo y se utiliza principalmente para visibilizar y 
compartir las investigaciones, discutir la investigación y facilitar la colaboración entre investigadores.

Finalmente, Sci-Hub https://sci-hub.tw/, un portal controvertido por democratizar el acceso y distribución del conocimiento científico al proporcionar acceso público y gratuito al texto completo de casi la totalidad de la literatura científica mundial, incluyendo artículos que se encuentran tras muro de pago o paywall $(36,37)$.

\section{Referencias}

1. Shuttleworth M, Wilson LT. Definition of Research [Internet]. [cited 2018 Dec 6]. Available from: https://explorable.com/definition-of-research

2. Rajasekar S, Philominathan P, Chinnathambi V. Research Methodology [Internet]. 2006 [cited 2018 Dec 6]. Available from: http://arxiv.org/abs/physics/0601009

3. Campos-Asensio C. How to develop a bibliographic search strategy. Enferm Intensiva. 2018 Oct;29(4):182-6.

4. Digital Object Identifier System [Internet]. [cited 2018 Dec 6]. Available from: https://www.doi.org/

5. El DOI, Identificador de Objetos Digitales. Inf tecnol. 2011;22(6):1-1.

6. UKSG. The DOI (Digital Object Identifier) [Internet]. [cited 2018 Dec 6]. Available from: https://www.uksg.org/serials/doi

7. F1000Research - An innovative open access publishing platform offering immediate publication and open peer review [Internet]. [cited 2018 Dec 6]. Available from: https://f1000research.com/

8. PeerJ [Internet]. [cited 2018 Dec 6]. Available from: https://peerj.com/

9. National Library of Medicine - National Institutes of Health [Internet]. U.S. National Library of Medicine. 1993 [cited 2018 Dec 6]. Available from: https://www.nlm.nih.gov

10. MEDLINE: Description of the Database [Internet]. U.S. National Library of Medicine. 2018 [cited 2018 Dec 6]. Available from: https://www.nlm.nih.gov/bsd/medline.html

11. OPAS-OMS. BIREME [Internet]. [cited 2018 Dec 6]. Available from: https://www.paho.org/bireme/index.php?lang=es

12. Portal Regional da BVS [Internet]. [cited 2018 Dec 6]. Available from: http://bvsalud.org/es/ 
13. LILACS - Literatura Latinoamericana y del Caribe en Ciencias de la Salud [Internet]. [cited 2018 Dec 6]. Available from: http://lilacs.bvsalud.org/es/

14. MeSH (Medical Subject Headings) [Internet]. National Library of Medicine (NLM) National Center for Biotechnology Information (NCBI). [cited 2018 Dec 6]. Available from: https://www.ncbi.nlm.nih.gov/mesh

15. DeCS - Descriptores en Ciencias de la Salud [Internet]. Biblioteca Virtual en Salud. [cited 2018 Dec 6]. Available from: http://decs.bvs.br/E/decsweb2018.htm

16. Research Preprints: Server List [Internet]. Google Docs. [cited 2018 Dec 26]. Available from:

https://docs.google.com/spreadsheets/d/17RgfuQcGJHKSsSJwZZn0oiXAnimZu2sZsWp8Z 6ZaYYo/edit?usp=embed_facebook

17. Authorea [Internet]. [cited 2018 Dec 26]. Available from: https://www.authorea.com/

18. arXiv.org e-Print archive [Internet]. [cited 2018 Dec 7]. Available from: https://arxiv.org

19. bioRxiv.org - the preprint server for Biology [Internet]. [cited 2018 Dec 7]. Available from: https://www.biorxiv.org

20. The YODA Project |MedRxiv [Internet]. [cited 2018 Dec 26]. Available from: http://yoda.yale.edu/medrxiv

21. OSF Preprints [Internet]. [cited 2018 Dec 26]. Available from: https://osf.io/preprints/

22. Preprints - The Multidisciplinary Preprint Platform [Internet]. [cited 2018 Dec 26]. Available from: https://www.preprints.org/

23. SocArXiv [Internet]. [cited 2018 Dec 26]. Available from: https://osf.io/preprints/socarxiv/

24. Zenodo - Research. Shared [Internet]. [cited 2018 Dec 26]. Available from: https://zenodo.org/

25. Van Den Berge K, Hembach K, Soneson C, Tiberi S, Clement L, Love MI, et al. PeerJ Preprints - Table of Contents [Internet]. [cited 2018 Dec 26]. Available from: https://peerj.com/preprints/

26. Home : Nature Precedings [Internet]. [cited 2018 Dec 26]. Available from: http://precedings.nature.com/

27. JP-JMIR Preprints [Internet]. [cited 2018 Dec 26]. Available from: https://preprints.jmir.org

28. PrePubMed [Internet]. [cited 2018 Dec 7]. Available from: http://www.prepubmed.org

29. Fyfe A, Moxham N, McDougall-Waters J. The Secret History of the Scientific Journal [Internet]. 2015 [cited 2018 Dec 7]. Available from: https://arts.standrews.ac.uk/philosophicaltransactions/ 
30. McDougall-Waters, Julie, Noah Moxham, and Aileen Fyfe. Philosophical Transactions: 350 Years of Publishing at the Royal Society (1665 - 2015) [Internet]. 2014 [cited 2018 Dec 7]. Available from: https://royalsociety.org/ /media/publishing350/publishing350-exhibitioncatalogue.pdf

31. U.S. National Library of Medicine. Key MEDLINE Indicators [Internet]. MEDLINE/PubMed Resources. 2002 [cited 2018 Dec 7]. Available from: https://www.nlm.nih.gov/bsd/bsd_key.html

32. Dirección General de Bibliotecas - UNAM. Cómo encontrar información [Internet]. Estrategias de búsqueda. 2016 [cited 2018 Dec 7]. Available from: http://biblio.unam.mx:8130/index.php/estrategias-de-busqueda

33. Unpaywall [Internet]. [cited 2018 Dec 7]. Available from: https://unpaywall.org

34. DOAJ. Directory of Open Access Journals [Internet]. [cited 2018 Dec 7]. Available from: https://doaj.org

35. Directory of Open Access Repositories - SHERPA Services [Internet]. [cited 2018 Dec 7]. Available from: http://v2.sherpa.ac.uk/opendoar/

36. Himmelstein DS, Romero AR, Levernier JG, Munro TA, McLaughlin SR, Greshake Tzovaras B, et al. Sci-Hub provides access to nearly all scholarly literature. Elife [Internet]. 2018 Mar 1;7. Available from: http://dx.doi.org/10.7554/eLife.32822

37. Till BM, Rudolfson N, Saluja S, Gnanaraj J, Samad L, Ljungman D, et al. Who is pirating medical literature? A bibliometric review of 28 million Sci-Hub downloads. Lancet Glob Health. 2019 Jan;7(1):e30-1. 